\title{
2-吡喃酮参与的不对称反电子需求 Diels-Alder 反应
}

\author{
丁祥峰䚮卫平*
}

(华东理工大学药学院 上海市新药设计重点实验室 上海 200237)

\section{Asymmetric Inverse-Electron-Demand Diels-Alder Reaction of 2-Pyrones

\author{
Ding, Xiangfeng Deng, Weiping* \\ (School of Pharmacy and Shanghai Key Laboratory of New Drug Design, East China University of \\ Science and Technology, Shanghai 200237)
}

手性 $c i s$-十氢䒺结构作为一类独特的全碳骨架, 是 许多药物以及活性天然产物分子的关键结构单元 ${ }^{[1]}$. 这些分子通常具有多个连续的、高度官能团化的手性中 心, 从而表现出结构以及生物活性的多样性. 因此, 设 计高效新颖的立体选择性合成该类骨架的策略, 引起了 研究人员的巨大兴趣 ${ }^{[2]}$. 目前, 构建十氢萗骨架的策略 主要可分为以下四类: (1) Wieland-Miescher 型酮参与的 Robinson 环化反应; (2)分子内 Diels-Alder 反应; (3)开链 双烯体与六元环亲双烯体的正电子需求 Diels-Alder 反 应; (4) Cope 重排反应. 但是考虑到含有 $c i s$-十氢荎骨架 天然产物结构的多样性, 发展高效、高立体选择性的新 型合成方法, 用于构建高度官能团化且带有多个手性中 心的 cis-十氢萗结构, 仍具有非常重要的研究意义.

Diels-Alder 反应已被证明是合成六元环的有效的 方法之一, 具有较高的化学选择性、区域选择性和立体 选择性 ${ }^{[3]}$. 通过逆合成分析, cis-十氢萘骨架可以由缺电 子双烯体与富电子环状共轭二烯通过反电子需求 DielsAlder 反应直接构建. 然而, 富电子的环已二烯通常作 为一类良好的双烯体, 与缺电子亲双烯体发生正电子需 求 Diels-Alder 反应 ${ }^{[4]}$. 目前该类化合物作为亲双烯体参 与反电子需求 Diels-Alder 反应尚未见报道. 2-吡喃酮作 为一类特殊的双烯体参与的 Diels-Alder 反应在复杂天 然产物合成中有着广泛的应用 ${ }^{[5]}$. 在此基础之上, 复旦 大学化学系蔡泉课题组设想使用缺电子 2-吡喃酮作为 双烯体, 富电子环已二烯作为亲双烯体, 通过 Lewis 酸 催化的不对称 Diels-Alder 反应构建 cis-十氢萗骨架.

近日, 该研究团队在 $\mathrm{Yb} / \mathrm{BINOL}$ 络合物的催化下,

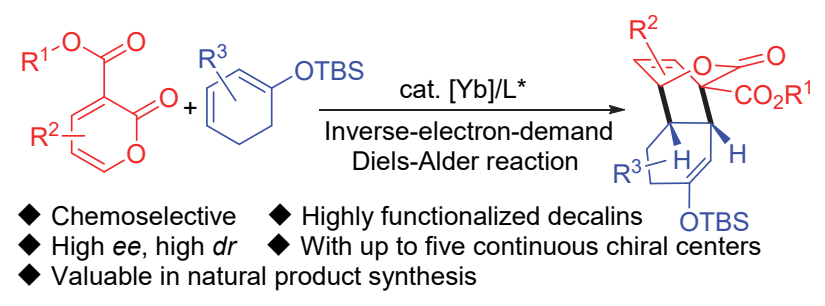

图 12 -吡喃酮参与的不对称反电子需求 Diels-Alder 反应 Figure 1 Asymmetric inverse-electron-demand Diels-Alder reaction of 2-pyrones

发展了2-吡喃酮衍生物与环己二烯醇硅醚衍生物的不 对称反电子需求 Diels-Alder 反应 ${ }^{[6]}$. 该方法能够以良好 的产率和立体选择性地合成了一系列高度官能团化的 $c i s-$ 一氢荎衍生物, 且条件温和、立体选择性高, 对不同 取代的 2-吡喃酮以及硅基环己二烯醇醚都具有良好的 兼容性. 值得注意的是, 通过调节手性环己二烯醇硅醚 $(R$ 或 $S)$ 以及配体 $(R$ 或 $S)$ 的绝对构型, 能够以优异的产 率、良好的非对映和对映选择性实现具有多达五个连续 手性中心的 $c i s-$ 一氢荎骨架化合物的立体发散性合成.

为了进一步体现该方法的应用价值, 研究人员通过 七步反应, 以 $31 \%$ 的总收率实现了 4-amorphen-11-ol 的 不对称全合成. 此外, 借助立体发散性合成策略, 研究 人员通过七步反应，首次实现了cis-crotonin的不对称全 合成，进一步说明了该反应的应用前景.

蔡泉课题组基于经典的 Diels-Alder 反应，使用 $\mathrm{Yb}(\mathrm{OTf})_{3} /$ 取代 BINOL 络合物作为催化剂, 成功实现了 2-吡喃酮衍生物与环己二烯醇硅醚衍生物之间的不对 称反电子需求 Diels-Alder 反应, 并以良好的产率和优秀

\footnotetext{
* Corresponding author. E-mail: weiping_deng@ecust.edu.cn. Published online October 5, 2020.
} 
<smiles></smiles>

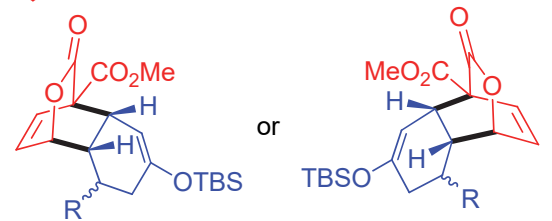

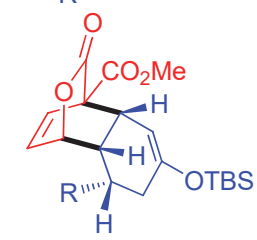

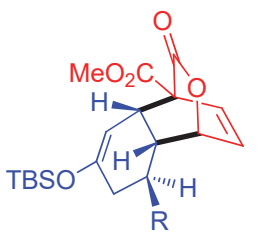

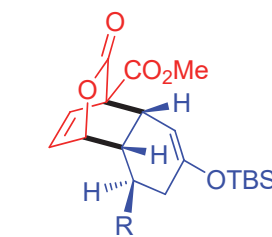

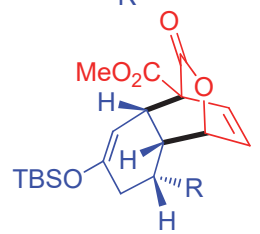

图 2 cis-十氢萗骨架的立体发散合成

Figure 2 Stereodivergent synthesis of substituted cis-decalin scaffold

的立体选择性获得一系列 cis-十氢荟衍生物. 此外, 通 过简单地改变配体和底物的绝对构型, 实现了各种取代 $c i s$-十氢萗化合物的立体发散性合成，并且基于该策略 实现了 4-amorphen-11-ol 以及 cis-crotonin 的不对称全合 成，具有很好的应用前景.
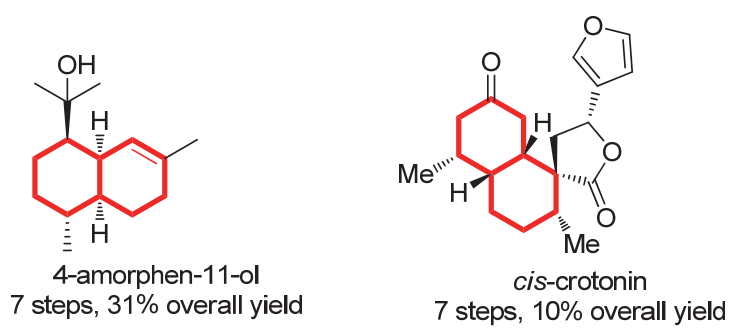

图 3 4-Amorphen-11-ol 以及 cis-crotonin 对映选择性全合成

Figure 3 Enantioselective total synthesis of 4-amorphen-11-ol and cis-crotonin

\section{References}

[1] Schnermann, M. J.; Shenvi, R. A. Nat. Prod. Rep. 2015, 32, 543.

[2] Dhambri, S.; Mohammad, S.; Nguyen Van Buu, O.; Galvani, G.; Meyer, Y.; Lannou, M.-I.; Sorin, G.; Ardisson, J. Nat. Prod. Rep. 2015, 32, 841 .

[3] Jiang, X.; Wang, R. Chem. Rev. 2013, 113, 5515.

[4] Earley, W. G.; Jacobsen, J. E.; Madin, A.; Meier, G. P.; O’Donnell, C. J.; Oh, T.; Old, D. W.; Overman, L. E.; Sharp, M. J. J. Am. Chem. Soc. 2005, 127, 18046.

[5] Cai, Q. Chin. J. Chem. 2019, 37, 946.

[6] Si, X.-G.; Zhang, Z.-M.; Zheng, C.-G.; Li, Z.-T.; Cai, Q. Angew. Chem. Int. Ed. 2020, 59, 18412. 